\title{
Incorporation of Urinary Neutrophil Gelatinase- Associated Lipocalin and Computed Tomography Quantification to Predict Acute Kidney Injury and In-Hospital Death in COVID-19 Patients
}

\author{
Li He $^{\mathrm{a}}$ Qunzi Zhang ${ }^{\mathrm{a}}$ Ze Li ${ }^{\mathrm{a}}$ Li Shen ${ }^{\mathrm{b}}$ Jiayin Zhang ${ }^{\mathrm{c}}$ Peng Wang ${ }^{\mathrm{d}}$ \\ Shan Wu ${ }^{e}$ Ting Zhou ${ }^{a}$ Qiuting $X^{a}{ }^{a}$ Xiaohua Chen ${ }^{d}$ Xiaohong Fan $^{f}$ \\ Ying Fan ${ }^{\mathrm{a}}$ Niansong Wang ${ }^{\mathrm{a}}$ \\ a'Department of Nephrology, Shanghai Jiao Tong University Affiliated Sixth People's Hospital, Shanghai, China; \\ ${ }^{b}$ Clinical Research Center, Shanghai Jiao Tong University Affiliated Sixth People's Hospital, Shanghai, China; \\ 'Department of Radiology, Shanghai Jiao Tong University Affiliated Sixth People's Hospital, Shanghai, China; \\ dDepartment of Infection, Shanghai Jiao Tong University Affiliated Sixth People's Hospital, Shanghai, China; \\ e'Department of Endoscopy, Shanghai Jiao Tong University Affiliated Sixth People's Hospital, Shanghai, China; \\ fDepartment of Pneumology, Shanghai Jiao Tong University Affiliated Sixth People's Hospital, Shanghai, China
}

\section{Keywords}

COVID-19 · Acute kidney injury · Urinary neutrophil gelatinase-associated lipocalin · In-hospital death . Artificial intelligence

\begin{abstract}
Background: The prevalence of acute kidney injury (AKI) in COVID-19 patients is high, with poor prognosis. Early identification of COVID-19 patients who are at risk for AKI and may develop critical illness and death is of great importance. $\mathbf{O b}$ jective: The aim of this study was to develop and validate a prognostic model of AKI and in-hospital death in patients with COVID-19, incorporating the new tubular injury biomarker urinary neutrophil gelatinase-associated lipocalin (uNGAL) and artificial intelligence (AI)-based chest computed tomography (CT) analysis. Methods: A single-center cohort of patients with COVID-19 from Wuhan Leishenshan Hospital were included in this study. Demographic characteristics, laboratory findings, and Al-assisted chest CT imaging variables identified on hospital admission were screened using
\end{abstract}

karger@karger.com www.karger.com/kdd

Karger ${ }^{\prime \prime} \div$

BOPEN ACCESS
(C) 2020 The Author(s)

Published by S. Karger AG, Basel

This article is licensed under the Creative Commons AttributionNonCommercial-NoDerivatives 4.0 International License (CC BYNC-ND) (http://www.karger.com/Services/OpenAccessLicense) Usage and distribution for commercial purposes as well as any distribution of modified material requires written permission. least absolute shrinkage and selection operator (LASSO) and logistic regression to develop a model for predicting the AKI risk. The accuracy of the AKI prediction model was measured using the concordance index (C-index), and the internal validity of the model was assessed by bootstrap resampling. A multivariate Cox regression model and Kaplan-Meier curves were analyzed for survival analysis in COVID-19 patients. $\boldsymbol{R e}$ sults: One hundred seventy-four patients were included. The median $( \pm S D)$ age of the patients was $63.59 \pm 13.79$ years, and 83 (47.7\%) were men. u-NGAL, serum creatinine, serum uric acid, and CT ground-glass opacity (GGO) volume were independent predictors of $\mathrm{AKI}$, and all were selected in the nomogram. The prediction model was validated by internal bootstrapping resampling, showing results similar to those obtained from the original samples (i.e., $0.958 ; 95 \% \mathrm{Cl}$ 0.9097-0.9864). The C-index for predicting AKI was 0.955 (95\% Cl 0.916-0.995). Multivariate Cox proportional hazards regression confirmed that a high u-NGAL level, an increased GGO volume, and lymphopenia are strong predictors of a

Li He, Qunzi Zhang, and Ze Li contributed equally to this study. 
poor prognosis and a high risk of in-hospital death. Conclusions: This model provides a useful individualized risk estimate of AKI in patients with COVID-19. Measurement of uNGAL and Al-based chest CT quantification are worthy of application and may help clinicians to identify patients with a poor prognosis in COVID-19 at an early stage.

(C) 2020 The Author(s)

Published by S. Karger AG, Basel

\section{Background}

In early December 2019, COVID-19 cases emerged in Wuhan, Hubei Province, China, and soon rapidly spread worldwide $[1,2]$. Recent studies indicate that $5-20 \%$ of COVID-19 patients develop a critical illness that is primarily characterized by acute respiratory distress syndrome, with a high mortality [3-5]. Although the initial clinical sign for the detection of COVID-19 is pneumonia, the virus can affect multiple organs and, among these, the kidney is one of the most susceptible organs involved [6]. The incidence of AKI in patients with COVID-19 infection ranges from 3 to 15\% worldwide [7] and may represent a crucial prognostic factor for death [8]. The traditional evaluation of AKI relies on urine output and serum creatinine (sCr) levels [9]; however, these are only indicators of established kidney damage, and changes in $\mathrm{sCr}$ or urinary output are neither sensitive nor specific for AKI [10]. Therefore, early identification of COVID-19 patients who are at risk for AKI and may develop a critical illness and death is of great importance.

Much attention should be focused on novel biomarkers, especially markers for acute tubular damage. Neutrophil gelatinase-associated lipocalin (NGAL) is a marker for distal tubular injury that is increased when tubular cells are structurally damaged and it is an early biomarker for septic, ischemic, or nephrotoxic harm to the kidney [11]. Urinary NGAL (u-NGAL) has been identified as a new biomarker for the diagnosis and evaluation of AKI [12]. It has also been indicated that u-NGAL exhibits a good performance in predicting mortality in AKI patients [12]. In addition, NGAL serves as an acute-phase protein that is increased in many human diseases, particularly infection, inflammation, and ischemia [13].

Chest computed tomography (CT) has been used as the first-line imaging modality for prompt diagnosis and disease monitoring during the course of COVID-19 pneumonia [14]. Typical imaging features of COVID-19 pneumonia are multifocal bilateral ground-glass opacities (GGO) with patchy consolidations. The GGO quick- ly expand, followed by disease progression, and the extent of involved pulmonary lesions has been correlated with adverse clinical results $[15,16]$. Artificial intelligence (AI) is a new medical technique employed for quantitative evaluation of pulmonary diseases, allowing a more precise quantification of pulmonary lesions based on different attenuation thresholds [17]. However, AI-based CT quantification has not been widely applied in clinical practice for several objective reasons, which has hampered the precise assessment of disease severity and prognosis in patients with COVID-19.

In our study, we aimed to determine the clinical characteristic and imaging features of AKI patients with COVID-19 infection admitted to Wuhan Leishenshan Hospital. This is the first study to examine whether u-NGAL, a new biomarker for renal tubular injury, is more sensitive and accurate in detecting patients with AKI and predicting renal outcomes than traditional renal function indexes. In addition, we developed and validated a prognostic model of AKI and in-hospital death in patients with COVID-19, incorporating u-NGAL, laboratory findings, and AI-based quantification of chest CT scans. This study may help clinicians to identify COVID-19 patients who are susceptible to AKI and a poor prognosis at an early stage.

\section{Materials and Methods}

\section{Patient Population}

Patients with COVID-19 admitted to Wuhan Leishenshan Hospital from February 10, 2020, to April 9, 2020, were enrolled into this study. Patients with the following conditions were excluded: acute hepatitis, tuberculosis, and a need for maintenance dialysis or renal transplantation. AKI was defined as an increase of $0.3 \mathrm{mg} / \mathrm{dL}$ sCr within $48 \mathrm{~h}$ or of $50 \%$ baseline sCr within 7 days based on Kidney Disease: Improving Global Outcomes (KDIGO) criteria [9]. Baseline sCr was defined as the sCr value on admission.

\section{Procedures}

The following patient characteristics were extracted on hospital admission: clinical signs and symptoms, laboratory data, demographic characteristics, medical history, treatment, and outcome data. The thresholds of these laboratory data were given by the testing laboratory in Wuhan Leishenshan Hospital. The upper limits of normal sCr in men and women are 104 and $84 \mathrm{mmol} / \mathrm{L}$, respectively. The upper limits of normal uric acid in men and women are 428 and $357 \mathrm{mmol} / \mathrm{L}$, respectively. The lower limit of blood lymphocyte counts was $1.1 \times 10^{9} / \mathrm{L}$. The estimated glomerular filtration rate (eGFR) was calculated using the Chronic Kidney Disease Epidemiology Collaboration (CKD-EPI) equation [18]. All data were confirmed by a team of experienced physicians.

\section{CT Findings}

All of the patients were examined using a 128-slice multidetector CT (Revolution Maxima; GE Medical Systems, Milwaukee, WI, 
Table 1. Clinical characteristics and outcomes of patients with COVID-19

\begin{tabular}{|c|c|c|c|c|}
\hline & $\begin{array}{l}\text { All } \\
(n=174)\end{array}$ & $\begin{array}{l}\text { Nonsevere } \\
(n=120)\end{array}$ & $\begin{array}{l}\text { Severe } \\
(n=54)\end{array}$ & $\begin{array}{l}p \text { value } \\
\text { (severe vs. } \\
\text { nonsevere) }\end{array}$ \\
\hline Age, years & $63.59 \pm 13.79$ & $60.48 \pm 13.34$ & $70.5 \pm 12.27$ & 0.000 \\
\hline Males & $83(47.7)$ & $50(41.7)$ & $33(61.1)$ & 0.018 \\
\hline Exposure to patients & $48(27.6)$ & $37(30.8)$ & $11(20.4)$ & 0.153 \\
\hline \multicolumn{5}{|l|}{ Comorbidities } \\
\hline Hypertension & $84(48.3)$ & $52(43.3)$ & $32(59.3)$ & 0.052 \\
\hline Diabetes & $51(29.3)$ & $29(24.2)$ & $22(40.7)$ & 0.027 \\
\hline Respiratory system disease & $16(9.2)$ & $6(5.0)$ & $10(18.5)$ & 0.010 \\
\hline Chronic kidney disease & $15(8.6)$ & $8(6.7)$ & $7(13)$ & 0.172 \\
\hline Coronary heart disease & $30(17.2)$ & $16(13.3)$ & $15(27.8)$ & 0.012 \\
\hline Cerebrovascular disease & $19(10.9)$ & $9(7.5)$ & $10(18.5)$ & 0.032 \\
\hline \multicolumn{5}{|l|}{ Complications } \\
\hline ARDS & $15(8.6)$ & $0(0)$ & $15(27.8)$ & 0.000 \\
\hline AKI & $20(11.5)$ & $1(0.8)$ & $19(35.2)$ & 0.000 \\
\hline Stage 1 & $11(6.3)$ & 0 & $11(20.4)$ & \\
\hline Stage 2 & $5(2.9)$ & $1(0.8)$ & $4(7.4)$ & \\
\hline Stage 3 & $4(2.3)$ & 0 & $4(7.4)$ & \\
\hline \multicolumn{5}{|l|}{ Clinical outcomes } \\
\hline Discharged from hospital & $155(89.1)$ & $120(0)$ & $35(64.8)$ & 0.000 \\
\hline Remained in hospital & $4(2.3)$ & 0 & $4(7.4)$ & 0.003 \\
\hline Death & $15(8.6)$ & 0 & $15(27.8)$ & 0.000 \\
\hline
\end{tabular}

Values are presented as medians \pm SD or numbers (\%). Severity was staged based on the guidelines for diagnosis and treatment of COVID-19 (trial 7th edition) published by the Chinese National Health Commission. ARDS, acute respiratory distress syndrome.

USA) in the supine position with inspiratory breath hold. AI-assisted lesion quantification was analyzed using commercially available software (LungDoc, version 1.19.1; ShuKun Network Technology, Beijing, China). The volumes of GGO and consolidation were automatically assessed based on different attenuation thresholds (-750 to $-300 \mathrm{HU}$ for GGO and -300 to $50 \mathrm{HU}$ for consolidation) [19].

Urinary NGAL and Microalbuminuria Levels

Urinary NGAL and microalbuminuria (MAU) levels were tested by Li He and Qiuting Xu using a bedside dry fluorescence immunoanalyzer (model tz-320; Relia, Jiangsu, China) according to the protocol provided by the manufacturer.

\section{Statistical Analyses}

We performed multiple imputation of missing patient clinical data. Normally distributed variables are described using means \pm $\mathrm{SD}$, and medians (IQR) were used for nonnormally distributed variables. Least absolute shrinkage and selection operator (LAS$\mathrm{SO})$ regression was used to decrease the potential collinearity of variables assessed from the same patient and overfitting of variables. We used a multivariate logistic regression model to calculate the OR and 95\% CI of powerful predictive factors influencing AKI. The model is presented as nomogram. receiver operating characteristic (ROC) curves were drawn, and the area under the curve (AUC) was calculated to compare the accuracy of the prediction model with traditional kidney function markers. The performance of the predictive model was also examined using a bootstrapping technique with 1,000 random sampling and replacement iterations. Harrell's C statistic was calculated with its 95\% CI to measure the predictive power of our model.

Univariable and multivariable Cox regression models were performed to estimate the HR and 95\% CI for risk factors associated with in-hospital death. ROC curves were used to determine cut-off points of u-NGAL levels and GGO volume that predicted all-cause mortality. Evaluation of differences in in-hospital mortality between subgroups of u-NGAL levels and GGO volume were assessed using a Kaplan-Meier survival curve with a log-rank test.

All statistical analyses were conducted using $\mathrm{R}$ version 4.0.0. Data processing and statistical analyses were conducted by an independent statistician. All tests were two-sided, and $p<0.05$ were considered statistically significant.

\section{Results}

\section{Patient Characteristics}

One hundred seventy-four patients with COVID-19 admitted to Wuhan Leishenshan Hospital from February 10, 2020, to April 9, 2020, were enrolled into our 
Table 2. Laboratory findings of patients with COVID-19

\begin{tabular}{llll}
$\begin{array}{l}\text { All } \\
(n=174)\end{array}$ & $\begin{array}{l}\text { Nonsevere } \\
(n=120)\end{array}$ & $\begin{array}{l}\text { Severe } \\
(n=54)\end{array}$ & $\begin{array}{l}p \text { value } \\
\text { (severe vs. } \\
\text { nonsevere) }\end{array}$ \\
\hline
\end{tabular}

Leucocyte count, $n \times 10^{9} / \mathrm{L}$

Lymphocyte count, $n \times 10^{9} / \mathrm{L}$

Neutrophil count, $n \times 10^{9} / \mathrm{L}$

Platelet count, $n \times 10^{9} / \mathrm{L}$

Hemoglobin, g/L

Erythrocyte sedimentation rate, $\mathrm{mm} / \mathrm{h}$

Albumin, g/L

Alanine aminotransferase, U/L

Aspartate aminotransferase, U/L

Lactic dehydrogenase, IU/L

Blood urea nitrogen, $\mathrm{nmol} / \mathrm{L}$

Serum creatinine, $\mu \mathrm{mol} / \mathrm{L}$

Peak serum creatinine, $\mu \mathrm{mol} / \mathrm{L}$

eGFR, $\mathrm{ml} / \mathrm{min} / 1.73 \mathrm{~m}^{2}$

$\mathrm{sUA}, \mu \mathrm{mol} / \mathrm{L}$

Cystatin C, mg/L

IL-6, pg/mL

IL-10, pg/mL

TNF- $\alpha, p g / m L$

C-reactive protein, $\mathrm{mg} / \mathrm{L}$

Procalcitonin, $\mathrm{ng} / \mathrm{mL}$

SAA, mg/L

u-NGAL, ng/mL

Microalbuminuria, $\mathrm{mg} / \mathrm{L}$

Urinary red blood cell count, $\mathrm{n} / \mu \mathrm{L}$

$\begin{aligned} 6.0 & (4.7-7.5) \\ 1.4 & (0.9-1.8) \\ 3.5 & (2.6-5.2) \\ 230.0 & (171.5-276.0) \\ 120.0 & (106.5-133.0) \\ 23.0 & (9.8-44.0) \\ 37.9 & (34.7-40.7) \\ 23.0 & (14.0-37.3) \\ 21.0 & (15.8-32.0) \\ 191.0 & (165.8-248.3) \\ 5.3 & (4.3-7.2) \\ 64.8 & (52.7-78.2) \\ 67.2 & (56.5-89.1) \\ 92.8 & (80.2-103.4) \\ 275.5 & (221.5-347.0) \\ 1.0 & (0.8-1.2) \\ 3.5 & (1.5-22.0) \\ 5.0 & (5.0-5.0) \\ 7.7 & (6.1-10.6) \\ 7.7 & (6.1-10.6) \\ 0.05 & (0.03-0.15) \\ 5.0 & (5.0-13.7) \\ 100.3 & (67.9-166.7) \\ 16.1 & (10.3-36.9) \\ 6.4 & (2.2-23.3) \\ & \end{aligned}$

$\begin{array}{cc}8.2(5.9-11.1) & 0.000 \\ 0.8(0.6-1.0) & 0.000 \\ 6.2(4.4-9.1) & 0.000 \\ 217.5(142.5-295.8) & 0.291 \\ 106.5(88.5-123.0) & 0.000 \\ 40.0(20.8-61.8) & 0.000 \\ 34.5(29.2-37.1) & 0.000 \\ 28.0(18.0-51.8) & 0.046 \\ 26.0(21.0-48.3) & 0.000 \\ 287.0(222.8-424.5) & 0.000 \\ 7.2(4.9-15.3) & 0.000 \\ 66.8(57.6-87.1) & 0.056 \\ 81.6(65.9-125.0) & 0.000 \\ 86.8(64.3-98.4) & 0.004 \\ 268.5(184.5-395.5) & 0.526 \\ 1.3(0.9-2.0) & 0.000 \\ 40.4(11.6-145.6) & 0.000 \\ 5.0(5.0-8.0) & 0.000 \\ 10.3(7.1-15.7) & 0.001 \\ 29.1(8.1-73.1) & 0.000 \\ 0.18(0.08-0.46) & 0.000 \\ 40.1(11.7-102.7) & 0.000 \\ 199.6(157.3-545.1) & 0.000 \\ 40.1(23.7-89.9) & 0.000 \\ 12.7(3.3-128.0) & 0.002\end{array}$

Data are presented as medians (IQR).

study. Table 1 shows the clinical characteristics and outcomes of patients with COVID-19. On hospital admission, 54 of 174 patients were considered to be severe, and the rest were considered to be nonsevere, according to the guidelines for diagnosis and treatment of COVID-19 published by NHC of China (6th Edition). Median patient age was 63 years, and $47.7 \%$ were male. Some patients, especially severe patients, presented with organ function injury, including 15 (8.6\%) with acute respiratory distress syndrome, 20 (11.5\%) with acute kidney injury (AKI), and 48 patients (27.6\%) had a history of exposure to those with COVID-19 (Table 1). In the end, 155 (89.1\%) patients were discharged from the hospital. The most common symptoms were fever $(60.9 \%)$, cough (63.2\%), and dyspnea (50.6\%) (online suppl. Table S1; for all online suppl. material, see www.karger. com/doi/10.1159/000511403). Among these patients, $171(98.3 \%)$ were treated with a high-flow nasal cannula, $32(18.3 \%)$ with mechanical ventilation, and $2(1.1 \%)$ with extracorporeal membrane oxygenation (ECMO) (online suppl. Table S1).

Compared to nonsevere patients, severe patients were more likely to exhibit lower lymphocyte counts (i.e., 0.8 and IQR $0.6-1.0$ vs. 1.6 and IQR $1.2-2.0 \times 10^{9} / \mathrm{L}$ ) and increased C-reactive protein (i.e., 29.1 and IQR 8.1-73.1 vs. 0.7 and IQR $0.5-3.1 \mathrm{mg} / \mathrm{L}$ ), erythrocyte sedimentation rates (i.e., 40.0 and IQR $20.8-61.8$ vs. 20.0 and IQR 9.0$32.0 \mathrm{~mm} / \mathrm{h}$ ), serum amyloid A (SAA) (i.e., 40.1 and IQR $11.7-102.7$ vs. 5.0 and IQR $5.0-8.5 \mathrm{mg} / \mathrm{L}$ ), and procalcitonin (i.e., 0.18 and IQR $0.08-0.46$ vs. 0.03 and IQR $0.03-$ $0.05 \mathrm{ng} / \mathrm{mL}$ ). In addition, severe patients tended to have higher liver enzyme levels and lower serum albumin levels (Table 2).

\section{Kidney Abnormalities, AKI, and In-Hospital Death}

During hospitalization, the incidence of AKI in the overall COVID-19 patient population who met the KDIGO criteria was $11.5 \%$, and it was significantly higher in 
Table 3. CT findings of patients with COVID-19

$\begin{array}{llll}\begin{array}{l}\text { All } \\ (n=174)\end{array} & \begin{array}{l}\text { Nonsevere } \\ (n=120)\end{array} & \begin{array}{l}\text { Severe } \\ (n=54)\end{array} & \begin{array}{l}p \text { value } \\ \text { (severe vs. } \\ \text { nonsevere) }\end{array}\end{array}$

\section{Semiquantitative evaluation}

Reticulation

Pleural effusion

Lymphadenopathy

Nodule

AI-assisted quantification

Total lesion volume, $\mathrm{cm}^{3}$

GGO volume, $\mathrm{cm}^{3}$

Consolidation volume, $\mathrm{cm}^{3}$

$\begin{array}{cc}90(60.8) & 44(46.8) \\ 44(29.7) & 11(11.7) \\ 11(7.3) & 5(5.3) \\ 6(6.3) & 1(1.1)\end{array}$

$98.7(30.4-294.4)$

$65.6(19.2-214.3)$

$3.5(0.8-16.1)$
$46(85.2)$

0.000

$33(61.1)$

$6(11.1)$

$5(9.3)$

0.000

0.205

0.016
$337.6(178.3-525.9)$

$227.4(106.1-388.0)$

$26.8(9.1-60.8)$
0.000

0.000

0.000

Data are presented as medians (IQR).

Table 4. Multivariate logistic regression model for predicting AKI in patients with COVID-19

\begin{tabular}{lrrrr}
\hline Variable & \multicolumn{1}{l}{ OR } & 95\% CI & $p$ value \\
\hline Intercept & -5.776 & 0.003 & & 0.000 \\
u-NGAL $(\mathrm{ng} / \mathrm{mL})$ & 0.005 & 1.005 & $1.002-1.008$ & 0.000 \\
$\mathrm{sUA}(\mu \mathrm{mol} / \mathrm{L})$ & 2.439 & 11.456 & $1.970-66.637$ & 0.007 \\
$\mathrm{sCr}(\mu \mathrm{mol} / \mathrm{L})$ & 2.305 & 10.020 & $1.609-62.401$ & 0.014 \\
$\mathrm{GGO}$ volume $\left(\mathrm{cm}^{3}\right)$ & 0.008 & 1.008 & $1.003-1.012$ & 0.000 \\
\hline
\end{tabular}

severe patients (35.2\%) than in nonsevere patients (1.0\%). Severe patients tended to exhibit kidney abnormalities, including higher peak sCr (i.e., 81.6 and IQR 65.9-125.0 vs. 64.8 and IQR $54.0-77.8 \mu \mathrm{mol} / \mathrm{L}$ ) and blood cystatin C levels (i.e., 1.3 and IQR $0.9-2.0$ vs. 0.9 and IQR $0.8-1.1$ $\mathrm{mg} / \mathrm{L}$ ) and increased u-NGAL levels (i.e., 199.6 and IQR $157.3-545.1$ vs. 79.0 and IQR $57.3-118.3 \mathrm{ng} / \mathrm{mL}$ ), urinary red blood cell counts (i.e., 12.7 and IQR 3.3-128.0 vs. 5.1 and IQR 1.7-14.5/ $\mu \mathrm{L}$ ) and MAU levels (i.e., 40.1 and IQR 23.7-89.9 vs. 13.1 and IQR $8.8-25.8 \mathrm{mg} / \mathrm{L}$ ) (Table 2). Inhospital death occurred in $8.6 \%$ of the patients (Table 1 ). The incidence of in-hospital death in patients with AKI was $25 \%$, which was significantly higher than in those without AKI (6.49\%).

\section{Chest CT Findings in COVID-19 Patients}

CT semiquantitative data revealed that severe patients had a higher incidence of pleural effusion (61.1 vs. 11.7\%), nodules (9.3 vs. $1.1 \%$ ), lymphadenopathy ( 11.1 vs. $5.3 \%$ ), and reticulation ( 85.2 vs. $46.8 \%$ ) compared to nonsevere patients. In terms of CT AI-assisted quantification, GGO and consolidation were 2 primary findings of the initial chest $\mathrm{CT}$, and severe patients had a larger GGO volume (i.e., 227.4 and IQR 106.1-388.0 vs. 36.9 and IQR 9.1-91.4 $\mathrm{cm}^{3}$ ), as well as a larger consolidation volume (i.e., 26.8 and IQR 9.1-60.8 vs. 1.5 and IQR $0.4-3.8 \mathrm{~cm}^{3}$ ), compared to nonsevere patients. (Table 3 ).

\section{Risk Factors Predicted AKI}

Variables measured on hospital admission were included in the LASSO regression. After LASSO regression selection, 4 variables remained significant predictors of AKI, including u-NGAL, sCr, serum uric acid (sUA), and GGO volume (Fig. 1). Upon inclusion of these 4 variables in a logistic regression model, they remained statistically significant predictors of AKI and were included in the prediction model as follows: $\mathrm{u}-\mathrm{NGAL}(\mathrm{OR}=1.005 ; 95 \%$ CI 1.002-1.008; $p=0.000)$, $\mathrm{sCr}(\mathrm{OR}=10.020 ; 95 \% \mathrm{CI}$ $1.609-62.401 ; p=0.014)$, sUA $(\mathrm{OR}=11.456 ; 95 \% \mathrm{CI}$ $1.970-66.637 ; p=0.007)$, and GGO volume (OR = 1.008; 95\% CI 1.003-1.012; $p=0.000$; Table 4).

\section{Prognostic Nomogram for AKI}

Since logistic regression analysis identified u-NGAL, GGO volume, sCr level, and sUA level as independent predictors, we developed a nomogram to quantitatively 


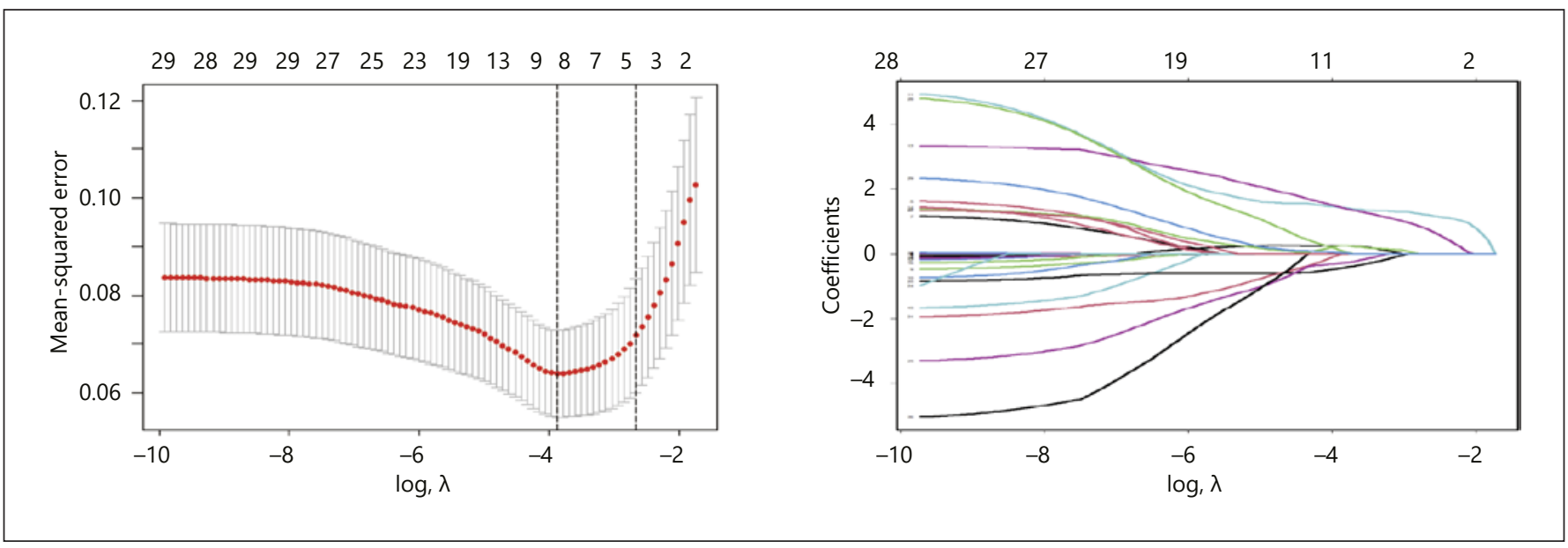

Fig. 1. Texture feature selection using the LASSO binary logistic regression model. a Tuning parameter $(\lambda)$ selection in the LASSO model used 10 -fold cross-validation via minimum criteria. The AUC for the ROC curve was plotted versus $\log (\lambda)$. Dotted vertical lines were drawn at the optimal values using the minimum criteria and the 1 SE of the minimum criteria (the 1-SE criteria). A $\lambda$ value of 0.076 , with a $\log (\lambda)$ of 2.567 was chosen (1-SE criteria) according to 10 -fold cross-validation. b LASSO coefficient profiles. A coefficient profile plot was produced against the $\log (\lambda)$ sequence. A vertical line was drawn at the value selected using 10 -fold crossvalidation, where the optimal $l$ resulted in 4 factors.

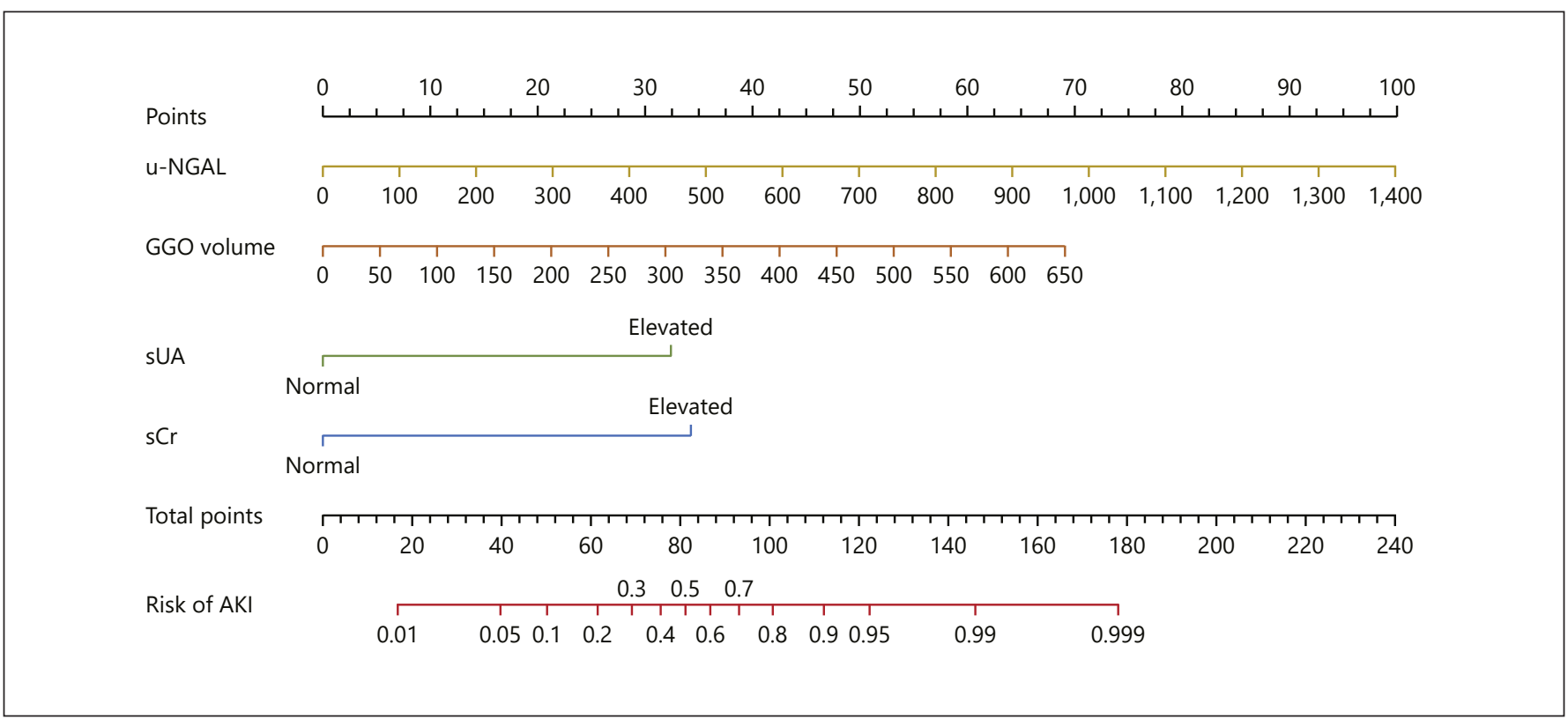

Fig. 2. Nomogram for the prediction of AKI based on a multivariable model. The nomogram allows users to obtain the risk of AKI corresponding to a patient's combination of variables. Points are assigned for each predictor by drawing a straight line upward from the corresponding value to the "points" line. Then, the points for each of the predictors are added, and the number is located on the "total points" axis. A line drawn straight down leads to the patient's probability of AKI. Normal representations of UA or sCr levels were within the thresholds of the laboratory. Elevated representations of UA or sCr levels were within the upper limits of the normal range. 
Table 5. Performance of the predictive model and kidney-related indicators for diagnosis of AKI in COVID-19 patients

\begin{tabular}{lllllll}
\hline Variable & AUC & 95\% CI & $p$ value & $\begin{array}{l}\text { Accuracy, } \\
\%\end{array}$ & $\begin{array}{l}\text { Sensitivity, } \\
\%\end{array}$ & $\begin{array}{l}\text { Specificity, } \\
\%\end{array}$ \\
\hline Model & 0.955 & $0.916,0.995$ & 0.000 & 95.40 & 95.0 & 85.1 \\
u-NGAL, ng/mL & 0.893 & $0.825,0.962$ & 0.000 & 91.37 & 80.0 & 92.9 \\
BUN, $\mathrm{mmol} / \mathrm{L}$ & 0.867 & $0.776,0.957$ & 0.000 & 87.93 & 65.0 & 90.9 \\
sUA, $\mu \mathrm{mol} / \mathrm{L}$ & 0.714 & $0.550,0.878$ & 0.002 & 89.66 & 55.0 & 94.2 \\
sCr, $\mu \mathrm{mol} / \mathrm{L}$ & 0.762 & $0.633,0.892$ & 0.000 & 91.95 & 50.0 & 97.4 \\
\hline
\end{tabular}

Table 6. Univariate and multivariate Cox analysis of clinical characteristics for the prognosis of in-hospital death

\begin{tabular}{|c|c|c|c|c|}
\hline \multirow[t]{2}{*}{ Variable } & \multicolumn{2}{|l|}{ Univariate analysis } & \multicolumn{2}{|l|}{ Multivariate analysis } \\
\hline & HR (95\% CI) & $p$ value & HR (95\% CI) & $p$ value \\
\hline u-NGAL (ng/mL) & $1.002(1.001-1.004)$ & 0.000 & $1.002(1.000-1.003)$ & 0.033 \\
\hline SAA (mg/L) & $20.713(2.722-157.594)$ & 0.003 & & \\
\hline GGO volume $\left(\mathrm{cm}^{3}\right)$ & $1.007(1.005-1.010)$ & 0.000 & $1.007(1.004-1.010)$ & 0.000 \\
\hline IL-10 (pg/mL) & $9.179(3.254-25.888)$ & 0.000 & & \\
\hline Lymphocyte count $\left(n \times 10^{9} / \mathrm{L}\right)$ & $0.077(0.017-0.341)$ & 0.001 & $0.145(0.032-0.659)$ & 0.012 \\
\hline $\mathrm{LDH}(\mathrm{IU} / \mathrm{L})$ & $10.265(2.890-36.466)$ & 0.000 & & \\
\hline
\end{tabular}

predict AKI after COVID-19 infection that included these independent predictors (Fig. 2).

\section{Performance Assessment and Internal Validation of the Nomogram}

The performance of the AKI model and renal function markers (blood urea nitrogen [BUN], sCr, sUA, and uNGAL) for identifying AKI patients among COVID-19 patients is described by ROC curves in online supplementary Figure S1. The AUC and 95\% CI for the diagnosis of AKI using BUN, sCr, sUA, and u-NGAL, as well as the prediction model, were 0.867 (95\% CI 0.776-0.957), 0.762 (95\% CI 0.633-0.892), 0.714 (95\% CI 0.550-0.878), 0.893 (95\% CI 0.825-0.962), and 0.955 (95\% CI 0.916-0.955), respectively. The AUC of AKI risk variables revealed that the prediction model exhibited a good diagnostic performance for detecting AKI $(p=0.000)$. The model was also demonstrated to have a higher accuracy (i.e., 0.95) with a sensitivity of 0.95 and a specificity of 0.85 compared to single renal function indicators (Table 5 ). The prediction model was validated by internal bootstrapping resampling, demonstrating that the results were similar to those obtained from the original samples (i.e., 0.958; 95\% CI 0.9097-0.9864). The nomogram had a C-index of 0.955 (95\% CI 0.916-0.995), suggesting a good discrimination ability.

\section{Risk Factors What Predict In-Hospital Death}

To investigate the risk factors that predict in-hospital death, variables measured on hospital admission were included in the LASSO regression. Six variables, including u-NGAL, lactate dehydrogenase (LDH), GGO volume, SAA, IL-10, and lymphocyte count, remained significant predictors of all-cause mortality after LASSO regression selection (online suppl. Fig. S2). After adjustment by univariate and multivariate Cox regression, the following 3 variables were still strongly associated with in-hospital death: u-NGAL levels (HR $=1.002$; 95\% CI 1.001-1.003; $p=0.033)$, GGO volume ( $\mathrm{HR}=1.007 ; 95 \%$ CI $1.004-$ $1.010 ; p=0.000)$, and lymphocyte count $(\mathrm{HR}=0.145 ; 95 \%$ CI $0.032-0.659 ; p=0.012$ ) (Table 6). ROC analysis revealed that the cut-off point of GGO volume that predicted in-hospital death was $214 \mathrm{~m}^{3}(\mathrm{AUC}=0.873)$. In the Kaplan-Meier analysis, we divided the patients into 2 groups based on the cut-off value. The group with a GGO volume $\geq 214 \mathrm{~cm}^{3}$ had a significantly higher in-hospital death rate than the group with a GGO volume $<214 \mathrm{~cm}^{3}$ (log-rank test; $p<0.001)$. According to the threshold value in our laboratory, the upper limit of u-NGAL levels was $150 \mathrm{ng} / \mathrm{mL}$, and the lower limit of blood lymphocyte counts was $1.1 \times 10^{9} / \mathrm{L}$. The group with u-NGAL levels $\geq 150 \mathrm{ng} / \mathrm{mL}$ had a significantly increased in-hospital death rate compared to the group with u-NGAL levels 


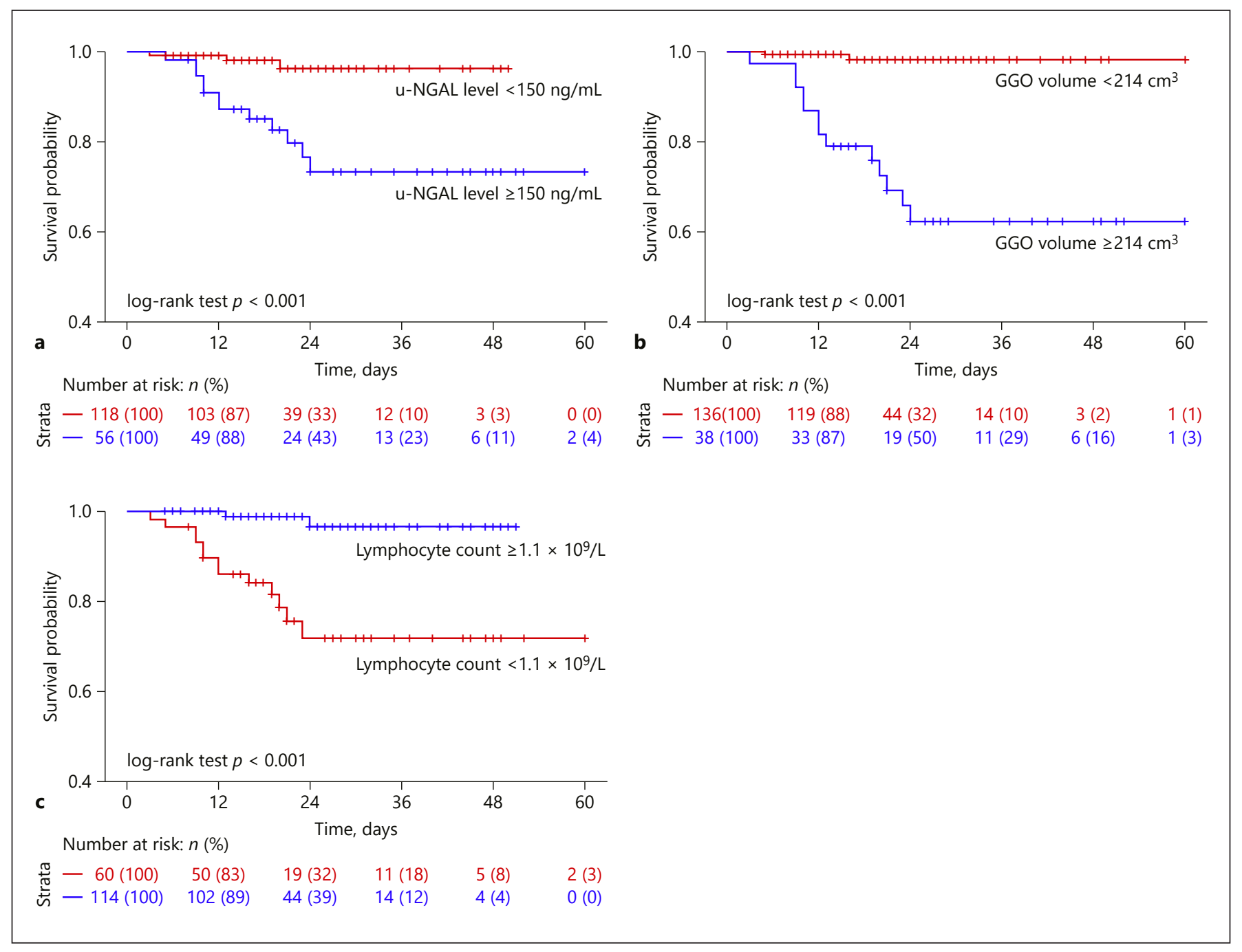

Fig. 3. Kaplan-Meier survival analysis. a u-NGAL level. b GGO volume. c Lymphocyte count. Comparison of curves using a log-rank test.

$<150 \mathrm{ng} / \mathrm{mL}$ (log-rank test; $p<0.001$ ). Patients with a lymphocyte count $<1.1 \times 10^{9} / \mathrm{L}$ had a significantly increased in-hospital death rate than patients with a lymphocyte count $\geq 1.1 \times 10^{9} / \mathrm{L}$ (log-rank test; $\left.p<0.001\right)$ (Fig. 3).

\section{Discussion}

In this single-center cohort study, we demonstrated a high prevalence of AKI (11.5\%) in hospitalized patients with COVID-19, which is consistent with previous studies $[2,3,20]$. AKI is an independent risk factor for mortality in COVID-19 patients [21]. The human kidney is a vulnerable target for SARS-CoV-2; however, how the kidney is affected by SARS-CoV-2 remains unclear [22]. The etiology of AKI in COVID-19 patients is likely to be multifactorial and diverse. Diao et al. [23] reported SARS$\mathrm{CoV}-2$ antigens aggregated in renal tubules and viral nucleocapsid protein retention in postmortem kidney samples, indicating that COVID-19 infects the human kidney tubules directly [24-25]. In an RNA-sequencing study, an up to 100 times higher tissue ACE2 expression in the kidneys was found compared to the lungs, suggesting that kidney injury may be induced by coronavirus in an ACE2dependent manner [26]. In addition to direct invasion of SARS-CoV-2, other secondary factors, particularly immune-mediated kidney damage, cytokine storm, hypox- 
ia, secondary infection with bacteria, other viruses, fungi, and drug-associated nephrotoxicity, can all lead to AKI [27-19].

Given the high prevalence and mortality of AKI in COVID-19 patients, it is urgent to identify novel biomarkers to enhance the diagnosis and prediction of AKI in the current battle against the SARS-CoV-2 virus. Although elevated $\mathrm{sCr}$ is useful for detecting changes in glomerular filtration, it often has a poor relationship with the onset of AKI or AKI progression [30, 31]. Furthermore, there exists a lag in time between acute tubular injury and upregulation of sCr in AKI patients [32]. NGAL is expressed at low levels in normal human tissues and it is rapidly released from renal tubular cells in response to various injurious stimuli, representing a novel, sensitive, and specific biomarker for early detection of AKI [3337]. Consistent with previous findings, based on our multivariate regression analysis and internal validation by a nomogram, we demonstrated that $\mathrm{u}$-NGAL represents an independent risk factor to predict AKI in patients with COVID-19, suggesting a crucial role of NGAL in the diagnosis and prediction of AKI in COVID-19 patients.

In addition, u-NGAL also proved to be a strong predictor of all-cause mortality in this study. There could be 2 possible reasons for this finding. First, high levels of $\mathrm{u}-$ NGAL indicate an abrupt loss of kidney function resulting in AKI, which is significantly correlated with increased mortality [9]. Second, u-NGAL may be involved in the inflammatory processes and it might be responsible for the lethal complications of SARS-CoV-2 infection [18, 38]. It has been reported that NGAL is an acute-phase protein likely to be elevated in many human diseases, particularly in the setting of inflammation, infection, and ischemia [9]. Recently, the magnitude of inflammatory storm was correlated with disease severity in COVID-19 patients [39]. Overwhelming proinflammatory cytokines, such as IL-6, IL-10, and SAA, not only cause pulmonary injury characterized by diffuse alveolar injury with endothelial and epithelial apoptosis but may also enter the systemic circulation, leading to extrapulmonary manifestations and multiple organ injury [40]. In our study, using LASSO regression analysis we found that inflammatory factors, such as IL-10, SAA, and lymphocyte count, were independent risk factors for in-hospital death, indicating that both a cytokine storm and lymphopenia are associated with poor in-hospital survival in COVID-19 patients, and the immune response may play an important role in directing disease progression [41, 42]. However, after adjustment by multivariate Cox regression, $\mathrm{u}-\mathrm{NGAL}$ remained a strong predictor associated with in-hospital death, suggesting dual crucial roles for NGAL in both AKI pathogenesis and a poor prognosis in COVID-19 patients.

In the current study, we also applied AI-assisted chest CT quantification for integrated prediction of disease progression. Chest CT is considered an important diagnostic approach in the management of COVID-19 infection for detection of pulmonary involvement [5]. However, conventional CT imaging only provides a visual diagnosis and is not able to precisely quantify pulmonary lesions. The latest technical development in the field of AI enables automatic lesion quantification using preset attenuation thresholds [43]. AI-based quantification was beneficial not only for disease diagnosis but also for prognosis evaluation. Studies have indicated that multiple GGO with consolidations in the periphery of the lungs comprise the basic CT feature in COVID-19 patients [44]. In our study, the GGO volume on the initial chest $\mathrm{CT}$ was proven to be an independent risk factor for the prediction of unfavorable clinical outcomes, including $\mathrm{AKI}$ and in-hospital death, suggesting that AI-assisted lesion quantification may represent a useful tool for risk stratification and prognosis evaluation in patients with COVID-19.

Here, for the first time, we developed an AKI prediction model combining the new biomarker u-NGAL, AIbased CT quantification of the GGO volume, and routine clinical biochemistry parameters. The heterogeneity of AKI indicated that multiple factors might be necessary for early detection and disease evaluation of AKI screening, as well as for optimizing the prognosis of AKI. Since the nomogram is a useful tool for medical decision-making [45], it can make the results of prediction models much clearer by showing the prediction visually. The current study is the first to incorporate 4 independent parameters (u-NGAL, GGO volume, sCr, and sUA) into a nomogram and develop risk scores, all of which are measurable and noninvasive. These prognostic scores could serve as a supplement for stratifying patients who are susceptible to AKI with COVID-19 infection. In addition, multivariate Cox analysis also showed that u-NGAL levels, GGO volume, and lymphopenia were the only 3 strong predictors that were independently associated with in-hospital death, suggesting the importance of $\mathrm{u}$ NGAL levels and GGO volume and that these factors may also be associated with a poor prognosis in COVID-19 patients.

Our study has several limitations. This is a single-center study with a limited sample size. Furthermore, clinical data for these patients after discharge was lacking, so we 
could not evaluate long-term outcomes. With a larger sample size, our model could be externally validated in an open and independent patient cohort.

In conclusion, this is the first study to develop and validate a noninvasive AKI prediction model incorporating the new biomarker u-NGAL and AI-based chest CT quantification to improve the detection and prognosis of AKI. Higher u-NGAL levels, an increased GGO volume and lymphopenia were all associated with increased inhospital mortality in COVID-19 patients. Measurement of u-NGAL and AI-based chest CT quantification are worthy of application in clinical practice for COVID-19 patients and hold promise for future clinical use.

\section{Statement of Ethics}

This study was approved by the institutional ethics committee for human research of the Shanghai Sixth People's Hospital (2020-KY-014[k]). This research study was conducted in accordance with the guidelines of the Declaration of Helsinki. All of the patients provided written informed consent.

\section{Conflict of Interest Statement}

All the authors declare no competing interests.

\section{Funding Sources}

This study was supported by the National Nature Science Foundation of China (81670657, 81870504, and 81870468), the Shanghai Jiao Tong University Gaofeng Talent Training Plan, and a clinical project (20192833).

\section{Author Contributions}

Y.F. and N.W. designed this research project. L.H., Q.Z., Q.X., P.W., X.F., S.W., and X.C. performed the experiments. L.H., Y.F., J.Z., Q.Z., Z.L., L.S., S.W., and T.Z. analyzed the data. L.H., Z.L., and Y.F. drafted this paper. Y.F. and N.W. edited and revised this paper. Y.F. and N.W. approved the final version of this paper.

\section{References}

1 Guan WJ, Ni ZY, Hu Y, Liang WH, Ou CQ, He JX, et al.; China Medical Treatment Expert Group for Covid-19. Clinical characteristics of coronavirus disease 2019 in China. N Engl J Med. 2020 Apr;382(18):1708-20.

2 Cecconi M, Piovani D, Brunetta E, Aghemo A, Greco M, Ciccarelli M, et al. Early Predictors of Clinical Deterioration in a Cohort of 239 Patients Hospitalized for Covid-19 Infection in Lombardy, Italy. J Clin Med. 2020 May;9(5):9.

3 Cummings MJ, Baldwin MR, Abrams D, Jacobson SD, Meyer BJ, Balough EM, et al. Epidemiology, clinical course, and outcomes of critically ill adults with COVID-19 in New York City: a prospective cohort study. Lancet. 2020 Jun;395(10239):1763-70.

4 Yang X, Yu Y, Xu J, Shu H, Xia J, Liu H, et al. Clinical course and outcomes of critically ill patients with SARS-CoV-2 pneumonia in Wuhan, China: a single-centered, retrospective, observational study. Lancet Respir Med. 2020 May;8(5):475-81.

5 World Health Organization. Coronavirus disease (COVID-2019) situation reports [Internet]. [cited 2020 Mar 16]. Available from: https://www.who.int/emergencies/diseases/ novelcoronavirus-2019/situation-reports.

6 Naicker S, Yang CW, Hwang SJ, Liu BC, Chen JH, Jha V. The Novel Coronavirus 2019 epidemic and kidneys. Kidney Int. 2020 May; 97(5):824-8.

7 Durvasula R, Wellington T, McNamara E, Watnick S. COVID-19 and kidney failure in the acute care setting: our experience from $\mathrm{Se}$ attle. Am J Kidney Dis. 2020;76(1):4-6.
8 Hirsch JS, Ng JH, Ross DW, Sharma P, Shah HH, Barnett RL, et al.; Northwell COVID-19 Research Consortium; Northwell Nephrology COVID-19 Research Consortium. Acute kidney injury in patients hospitalized with COVID-19. Kidney Int. 2020 Jul;98(1): 209-18.

9 Kellum JA, Lameire N; KDIGO AKI Guideline Work Group. Diagnosis, evaluation, and management of acute kidney injury: a KDIGO summary (Part 1). Crit Care. 2013 Feb; 17(1):204.

10 Doi K, Katagiri D, Negishi K, Hasegawa S, Hamasaki Y, Fujita T, et al. Mild elevation of urinary biomarkers in prerenal acute kidney injury. Kidney Int. 2012 Nov;82(10):1114-20.

11 Levey AS, James MT. Acute Kidney Injury. Ann Intern Med. 2017 Nov; 167(9):ITC66-80.

12 Westhoff JH, Seibert FS, Waldherr S, Bauer F, Tönshoff B, Fichtner A, et al. Urinary calprotectin, kidney injury molecule-1, and neutrophil gelatinase-associated lipocalin for the prediction of adverse outcome in pediatric acute kidney injury. Eur J Pediatr. 2017 Jun; 176(6):745-55.

13 Moschen AR, Adolph TE, Gerner RR, Wieser V, Tilg H. Lipocalin-2: A Master Mediator of Intestinal and Metabolic Inflammation. Trends Endocrinol Metab. 2017 May;28(5): 388-97.

14 Ai T, Yang Z, Hou H, Zhan C, Chen C, Lv W, et al. Correlation of Chest CT and RT-PCR Testing for Coronavirus Disease 2019 (COVID-19) in China: A Report of 1014 Cases. Radiology. 2020 Aug;296(2):E32-40.
15 Zu ZY, Jiang MD, Xu PP, Chen W, Ni QQ, Lu GM, et al. Coronavirus disease 2019 (COVID-19): A perspective from China. Radiology. 2020 Aug;296(2):E15-25.

16 Yang R, Li X, Liu H, Chest CT severity score: an imaging tool for assessing severe $\mathrm{CO}$ VID-19. Radiol Cardiothorac Imaging. 2020. 2(2):e200047.

17 Fischer AM, Varga-Szemes A, van Assen M, Griffith LP, Sahbaee P, Sperl JI, et al. Comparison of Artificial Intelligence-Based Fully Automatic Chest CT Emphysema Quantification to Pulmonary Function Testing. AJR Am J Roentgenol. 2020 May;214(5):1065-71.

18 Levey AS, Stevens LA, Schmid CH, Zhang YL, Castro AF 3rd, Feldman HI, et al.; CKD-EPI (Chronic Kidney Disease Epidemiology Collaboration). A new equation to estimate glomerular filtration rate. Ann Intern Med. 2009 May;150(9):604-12.

19 Kauczor HU, Heitmann K, Heussel CP, Marwede D, Uthmann T, Thelen M. Automatic detection and quantification of ground-glass opacities on high-resolution CT using multiple neural networks: comparison with a density mask. AJR Am J Roentgenol. 2000 Nov; 175(5):1329-34.

20 Cheng Y, Luo R, Wang K, Zhang M, Wang Z, Dong $L$, et al. Kidney disease is associated with in-hospital death of patients with COVID-19. Kidney Int. 2020 May;97(5):829-38.

21 Lim JH, Park SH, Jeon Y, Cho JH, Jung HY, Choi JY, et al. Fatal Outcomes of COVID-19 in Patients with Severe Acute Kidney Injury. J Clin Med. 2020 Jun;9(6):9. 
22 Perico L, Benigni A, Remuzzi G. Should COVID-19 Concern Nephrologists? Why and to What Extent? The Emerging Impasse of Angiotensin Blockade. Nephron. 2020;144(5): 213-21.

23 Diao B, Wang C, Tan Y, Chen X, Liu Y, Ning $\mathrm{L}$, et al. Reduction and functional exhaustion of $\mathrm{T}$ cells in patients with coronavirus disease 2019 (COVID-19). Front Immunol. 2020 May;11:827.

24 Pan XW, Xu D, Zhang H, Zhou W, Wang LH, Cui XG. Identification of a potential mechanism of acute kidney injury during the COVID-19 outbreak: a study based on single-cell transcriptome analysis. Intensive Care Med. 2020 Jun;46(6):1114-6.

25 Su H, Yang M, Wan C, Yi LX, Tang F, Zhu $\mathrm{HY}$, et al. Renal histopathological analysis of 26 postmortem findings of patients with COVID-19 in China. Kidney Int. 2020 Jul;98(1): 219-27.

26 Post A, Dullaart RP, Bakker SJ. Sodium status and kidney involvement during COVID-19 infection. Virus Res. 2020 Sep;286:198034.

27 Chu KH, Tsang WK, Tang CS, Lam MF, Lai $\mathrm{FM}$, To KF, et al. Acute renal impairment in coronavirus-associated severe acute respiratory syndrome. Kidney Int. $2005 \mathrm{Feb}$;67(2): 698-705.

28 Wang D, Hu B, Hu C, Zhu F, Liu X, Zhang J, et al. Clinical Characteristics of 138 Hospitalized Patients With 2019 Novel Coronavirus-Infected Pneumonia in Wuhan, China. JAMA. 2020 Feb;323(11):1061-9.

29 Kumar A, Zarychanski R, Pinto R, Cook DJ, Marshall J, Lacroix J, et al.; Canadian Critical Care Trials Group H1N1 Collaborative. Critically ill patients with 2009 influenza A(H1N1) infection in Canada. JAMA. 2009 Nov; 302(17):1872-9.
30 Al-Ismaili Z, Palijan A, Zappitelli M. Biomarkers of acute kidney injury in children: discovery, evaluation, and clinical application. Pediatr Nephrol. 2011 Jan;26(1):29-40.

31 Koyner JL, Garg AX, Coca SG, Sint K, Thiessen-Philbrook H, Patel UD, et al.; TRIBE-AKI Consortium. Biomarkers predict progression of acute kidney injury after cardiac surgery. J Am Soc Nephrol. 2012 May;23(5):905-14.

32 Greenberg JH, Zappitelli M, Jia Y, ThiessenPhilbrook HR, de Fontnouvelle CA, Wilson FP, et al. Biomarkers of AKI Progression after Pediatric Cardiac Surgery. J Am Soc Nephrol. 2018 May;29(5):1549-56.

33 Mishra J, Ma Q, Prada A, Mitsnefes M, Zahedi K, Yang J, et al. Identification of neutrophil gelatinase-associated lipocalin as a novel early urinary biomarker for ischemic renal injury. J Am Soc Nephrol. 2003 Oct;14(10): 2534-43.

34 Devarajan P. Neutrophil gelatinase-associated lipocalin-an emerging troponin for kidney injury. Nephrol Dial Transplant. 2008 Dec;23(12):3737-43.

35 Haase M, Bellomo R, Devarajan P, Schlattmann P, Haase-Fielitz A; NGAL Meta-analysis Investigator Group. Accuracy of neutrophil gelatinase-associated lipocalin (NGAL) in diagnosis and prognosis in acute kidney injury: a systematic review and meta-analysis. Am J Kidney Dis. 2009 Dec;54(6):1012-24.

36 Zhang A, Cai Y, Wang PF, Qu JN, Luo ZC, Chen XD, et al. Diagnosis and prognosis of neutrophil gelatinase-associated lipocalin for acute kidney injury with sepsis: a systematic review and meta-analysis. Crit Care. 2016 Feb;20(1):41.

37 Dewitte A, Joannès-Boyau O, Sidobre C, Fleureau C, Bats ML, Derache P, et al. Kinetic eGFR and Novel AKI Biomarkers to Predict Renal Recovery. Clin J Am Soc Nephrol. 2015 Nov;10(11):1900-10.
38 Warszawska JM, Gawish R, Sharif O, Sigel S, Doninger B, Lakovits K, et al. Lipocalin 2 deactivates macrophages and worsens pneumococcal pneumonia outcomes. J Clin Invest. 2013 Aug; 123(8):3363-72.

39 Liu J, Li S, Liu J, Liang B, Wang X, Wang H, et al. Longitudinal characteristics of lymphocyte responses and cytokine profiles in the peripheral blood of SARS-CoV-2 infected patients. EBioMedicine. 2020 May;55:102763.

40 Liu B, Li M, Zhou Z, Guan X, Xiang Y. Can we use interleukin-6 (IL-6) blockade for coronavirus disease 2019 (COVID-19)-induced cytokine release syndrome (CRS)? J Autoimmun. $2020 \mathrm{Jul} ; 111: 102452$.

41 Li X, Xu S, Yu M, Wang K, Tao Y, Zhou Y, et al. Risk factors for severity and mortality in adult COVID-19 inpatients in Wuhan. J Allergy Clin Immunol. 2020 Jul;146(1):110-8.

42 Wan S, Yi Q, Fan S, Lv J, Zhang X, Guo L, et al. Relationships among lymphocyte subsets, cytokines, and the pulmonary inflammation index in coronavirus (COVID-19) infected patients. Br J Haematol. 2020 May; 189(3): $428-37$.

43 Huang L, Han R, Ai T, Yu P, Kang H, Tao Q, et al. Serial quantitative chest CT assessment of COVID-19: deep-learning approach. Radiol Cardiothorac Imaging. 2020;2(2): e200075.

44 Zhou Z, Guo D, Li C, Fang Z, Chen L, Yang $\mathrm{R}$, et al. Coronavirus disease 2019: initial chest CT findings. Eur Radiol. 2020 Aug;30(8): 4398-406.

45 Jiang S, Yu T, Zhang Z, Fang J, Wang Y, Yang $\mathrm{Y}$, et al. Prognostic nomogram and score to predict renal survival of patients with biopsyproven diabetic nephropathy. Diabetes Res Clin Pract. 2019 Sep;155:107809. 